\title{
Exact results in four-dimensional Gauge Theories from Matrix models
}

\author{
Jorge Russo*† \\ Institució Catalana de Recerca i Estudis Avançats (ICREA) \\ Pg.Lluis Compayns, 23, 08010 Barcelona, Spain \\ Departament de Física Cuántica i Astrofísica and Institut de Ciències del Cosmos \\ Universitat de Barcelona, Martí Franquès, 1, 08028 Barcelona, Spain \\ E-mail: jorge.russo@icrea.cat
}

\begin{abstract}
We use localization and the Seiberg-Witten curve to exactly compute supersymmetric observables in different $\mathscr{N}=2$ four-dimensional theories. We first consider $\mathscr{N}=2$ SQCD with gauge group $S U(2)$ and two massive flavors. We compute the exact free energy, including all instanton contributions, and show that the theory undergoes a second-order quantum phase transition at a critical coupling. In the second part, we use localization to compute large $N$ correlation functions in superconformal field theories.
\end{abstract}

Corfu Summer Institute 2016 "School and Workshops on Elementary Particle Physics and Gravity" 31 August - 23 September, 2016

Corfu, Greece

\footnotetext{
*Speaker.

${ }^{\dagger}$ We thank D. Rodriguez-Gomez for collaboration in the second part of this work. This work is partly supported by projects FPA2013-46570, 2014-SGR-104 and MDM-2014-0369 of ICCUB (Unidad de Excelencia 'María de Maeztu').
} 


\section{Introduction}

I am honoured to be invited to speak at this School, dedicated to the memory of Ioannis Bakas. I had the chance to interact with Ioannis a number of times and enjoyed reading some of his many original contributions to theoretical physics. Ioannis was a very solid and eclectic theoretical physics, he wrote extremely interesting papers in many diverse topics such as string sigma models, large $N$ limit of extended conformal symmetries, $W$ algebras, non-associative structure in physics, integrable models, holography, WZW models, gauged supergravity, etc. Many of these papers led to new insights and new progresses. This work is dedicated to his memory.

There is now a large class of observables in different supersymmetric gauge theories in various dimensions that have been computed exactly. They can be used to explore fascinating aspects of gauge theories, such as dualities, large $N$ physics, resurgence, non-perturbative phenomena and integrable systems. A powerful tool to find exact results is supersymmetric localization, which has been used, in particular, to compute the partition function of general $\mathscr{N}=2$ four-dimensional theories, with any gauge group and matter content $[1,2,3]$. The result is expressed in terms of a finite dimensional integral, which is still difficult to compute. Here, using some examples, we will describe how to compute this integral in two interesting limits, the decompactification limit and the large $N$ limit. In both cases, we will show that the integral is exactly computable by the saddle-point method.

In the first part of this talk, we will discuss new results on the phase transition occurring in $\mathscr{N}=2$ SQCD with gauge group $S U(2)$ and two massive flavors of mass $M$, found in [4]. The theory depends on the coupling $\Lambda / M$. In [4], the free energy was computed in a strong coupling regime $\Lambda / M>2$. We will extend the results of [4] by finding the saddle point that dominates the integral in the weak coupling regime $\Lambda / M<2$. Combining both results, we will compute the free energy for any coupling and show that the second derivative has a discontinuity at $\Lambda=2 M$, implying a quantum phase transition of the second order.

The second part of the talk is based on the published works $[6,7]$ in collaboration with Diego Rodriguez-Gomez. We consider four-dimensional $\mathscr{N}=2$ superconformal gauge theories. We shall discuss correlation functions of chiral primary operators (CPO's) at large $N$, by using a method proposed in [5]. By extending this construction, we shall also show how to compute correlation functions of chiral primary operators and Wilson loops.

\section{2. $\mathscr{N}=2$ supersymmetric gauge theories}

Here we will study $\mathscr{N}=2$ supersymmetric gauge theories on $\mathbb{S}^{4}$ of radius $R$ with gauge group $U(N)$ or $S U(N)$. We shall consider the following field content:

Vector multiplet $\quad\left(A_{\mu}, \lambda_{\alpha}, \psi_{\alpha}, \Phi+i \Phi^{\prime}\right)$
Matter hypermultiplets $\quad\left(\phi, \chi_{\alpha}, \tilde{\chi}_{\alpha}, \tilde{\phi}\right) \quad$ (adjoint or fundamental)

The exact partition function for $\mathscr{N}=2$ supersymmetric Yang-Mills theories on $\mathbb{S}^{4}$, with arbitrary matter content, was computed by Pestun in [3]. The partition function is localized to an 
integral over the Coulomb moduli

$$
\langle\Phi\rangle=\operatorname{diag}\left(a_{1}, \ldots, a_{N}\right)
$$

The partition function for the $S U(N)$ theory is given by

$$
Z=\int \mathscr{D} a Z_{1-\text { loop }}\left|Z_{\text {inst }}(a)\right|^{2} e^{-S_{c l}}
$$

where

$$
\mathscr{D} a=d^{N-1} a \prod_{i<j}\left(a_{i}-a_{j}\right)^{2},
$$

$S_{c l}$ represents the classical action

$$
S_{c l}=\frac{1}{4 g_{Y M}^{2}} \int_{\mathbb{S}^{4}} d^{4} x \sqrt{g} R \operatorname{Tr} \Phi^{2}
$$

Thus the classical action localizes onto:

$$
S_{c l}=R^{2} \frac{8 \pi^{2}}{g_{Y M}^{2}} \sum_{i} a_{i}^{2}
$$

$Z_{1-\text { loop }}$ is expressed in terms of the function

$$
H(x) \equiv \prod_{n=1}^{\infty}\left(1+x^{2} / n^{2}\right) e^{-x^{2} / n},
$$

which constitutes the building block for the different multiplet contributions:

$$
\begin{array}{rlr}
Z_{1-\text { loop }} & \rightarrow \prod_{i<j}^{N} H^{2}\left(a_{i}-a_{j}\right) & \text { Vector multiplet } \\
& \rightarrow \prod_{i<j}^{N} \frac{1}{H\left(a_{i}-a_{j}+M\right) H\left(a_{i}-a_{j}-M\right)} & \text { Adjoint hypermultiplet } \\
& \rightarrow \prod_{i=1}^{N} \frac{1}{H\left(a_{i}+M\right)} & \text { (anti)Fundamental hypermultiplet }
\end{array}
$$

Finally, the instanton factor has the form:

$$
Z_{\text {inst }}=\sum_{k=0}^{\infty} q^{k} Z_{k}\left(M, a, \varepsilon_{1}, \varepsilon_{2}\right),
$$

for

$$
q=e^{2 \pi i \tau}, \quad \tau=\frac{\theta}{2 \pi}+\frac{4 \pi i}{g_{Y M}^{2}}, \quad \varepsilon_{1}=\varepsilon_{2}=1 / R,
$$

where $Z_{k}$ can be computed using the general Nekrasov construction $[1,2]$ with equivariant parameters $\varepsilon_{1}=\varepsilon_{2}=1 / R$. 


\section{Limits}

Computing the integral defining $Z$ is still very complicated. In four dimensions, only for the $\mathscr{N}=4$ theory the integral can be carried out exactly (in lower dimensions, there are many examples). In other $\mathscr{N}=2$ four-dimensional theories, we may compute the perturbation series by expanding the integrand. Alternatively, if we are interested in finding a closed analytic expression, we may consider limits:

- Large $N$, R arbitrary ( $\lambda=g^{2} N$ fixed). This implies two big simplifications that will allow us to determine $Z$ exactly:

a) At $N=\infty$ the integral is exactly determined by a saddle-point.

b) Instantons do not contribute, since $|q|=\left|e^{2 \pi i \tau}\right|=\left|e^{-8 \pi^{2} N / \lambda}\right| \rightarrow 0$.

- Finite $N$ (e.g. $S U(2)$ ) but $R \rightarrow \infty$ [4]. Then $Z$ is still exactly determined by a saddle point (this time the large parameter being $M R$ ). In this case, instantons contribute. They can be incorporated exactly by the Seiberg-Witten curve.

\section{3. $\mathscr{N}=2$ supersymmetric $S U(2)$ SQCD with two massive flavors}

\subsection{The partition function}

Consider now $\mathscr{N}=2$ supersymmetric Yang-Mills theory coupled to $N_{f}=2$ massive matter, namely a fundamental and an antifundamental hypermultiplet of mass $M$. This theory is asymptotically free. Localization now leads to the following partition function [4]

$$
Z^{\operatorname{SQCD}}\left(\mathbb{S}^{4}\right)=\text { const. } \int_{-\infty}^{\infty} d a a^{2} e^{4 a^{2} R^{2} \ln \Lambda R e^{1+\gamma}} \frac{H^{2}(2 a R)}{H^{2}\left(a R+\frac{M R}{\sqrt{2}}\right) H^{2}\left(a R-\frac{M R}{\sqrt{2}}\right)}\left|Z_{\text {inst }}(a)\right|^{2} .
$$

This integral is extremely complicated. Strikingly, it can be exactly determined in the decompactification limit, as shown below. This will give $F=-\ln Z^{\mathrm{SQCD}}$ as $F=F(\Lambda / M)$ in terms of elliptic functions. The possible phase transitions are determined by the discontinuities of $F$ that occur as the coupling $\Lambda / M$ is varied from 0 to infinity.

The decompactification limit implies looking into the infrared regime, where $\Lambda R \gg 1$. Since the integrand depends on $a R, \Lambda R$ and $\Lambda / M$, at large $\Lambda R$, we look for saddle-points dominating the integral lying at large $a R$. Thus we can use the asymptotic formula

$$
\ln H(x)=-x^{2} \ln |x| e^{\gamma-\frac{1}{2}}+O(\ln x) .
$$

In addition, we use the formula [1]

$$
2 \pi i \mathscr{F}_{\text {ins }}(a)=\lim _{\varepsilon_{1,2} \rightarrow 0} \varepsilon_{1} \varepsilon_{2} \ln Z_{\text {ins }} .
$$

We find

$$
\lim _{R \gg 1} Z^{\mathrm{SQCD}}\left(\mathbb{S}^{4}\right)=\int d a e^{-R^{2} S(a, M)},
$$


with

$$
\begin{aligned}
S(a, M) & =8 a^{2} \ln \frac{2 e^{\frac{1}{4}} a}{\Lambda}-2\left(a+\frac{M}{\sqrt{2}}\right)^{2} \ln \frac{\left|a+\frac{M}{\sqrt{2}}\right|}{\Lambda}-2\left(a-\frac{M}{\sqrt{2}}\right)^{2} \ln \frac{\left|a-\frac{M}{\sqrt{2}}\right|}{\Lambda} \\
& -2 \pi i \mathscr{F}_{\text {ins }}+2 \pi i \overline{\mathscr{F}}_{\text {ins }} .
\end{aligned}
$$

We recognize the one-loop contribution to the prepotential, which, combined with the instanton contributions, gives the full prepotential of the theory [8, 9]. The singularity at $a= \pm M / \sqrt{2}$ represents the point in the moduli space where the hypermultiplet becomes massless.

Note that the $\ln \Lambda$ piece originating from the curvature coupling of the scalar field of the vector multiplet has combined with the terms from the one-loop determinant to produce the correct oneloop terms of the prepotential with the dynamical scale $\Lambda$ included. Thus, denoting by $a^{*}$ the saddle-point dominating the integral, we get the exact partition function as $Z^{\mathrm{SQCD}}=Z^{\mathrm{SQCD}}(\Lambda / M)$,

$$
\lim _{R \rightarrow \infty} \frac{1}{R^{2}} \ln Z^{\mathrm{SQCD}}\left(\mathbb{S}^{4}\right)=2 \pi i \mathscr{F}\left(a^{*}\right)-2 \pi i \overline{\mathscr{F}}\left(a^{*}\right),
$$

The limit is taken with $M, \Lambda$ fixed. To complete the derivation, we need to incorporate the instantons in (3.5). This is done in the next subsection using the Seiberg-Witten formalism.

\subsection{Exact results via Seiberg-Witten}

The Seiberg-Witten curve for $\mathscr{N}=2 S U(2)$ SYM with two flavors of equal mass is [10]

$$
y^{2}=\left(x^{2}-\frac{1}{64} \Lambda^{4}\right)(x-u)+\frac{1}{4} M^{2} \Lambda^{2} x-\frac{1}{32} M^{2} \Lambda^{4} .
$$

The prepotential $\mathscr{F}(a)$ can then be obtained from the formula

$$
a_{D}=\frac{\partial \mathscr{F}}{\partial a} .
$$

where $a$ and $a_{D}$ are defined as period integrals of the meromorphic one-form

$$
\lambda=-\frac{\sqrt{2}}{4 \pi} \frac{y d x}{x^{2}-\frac{\Lambda^{4}}{64}} .
$$

This determines $a$ and $a_{D}$ in terms $u$ (see below).

By a shift $x \rightarrow x+u / 3$, we can write the curve (3.7) in the Weierstrass form

$$
y^{2}=\left(x-e_{1}\right)\left(x-e_{2}\right)\left(x-e_{3}\right),
$$

with

$$
\begin{aligned}
& e_{1}=\frac{u}{6}-\frac{\Lambda^{2}}{16}+\frac{1}{2} \sqrt{u+\frac{\Lambda^{2}}{8}+\Lambda M} \sqrt{u+\frac{\Lambda^{2}}{8}-\Lambda M} \\
& e_{2}=-\frac{u}{3}+\frac{\Lambda^{2}}{8} \\
& e_{3}=\frac{u}{6}-\frac{\Lambda^{2}}{16}-\frac{1}{2} \sqrt{u+\frac{\Lambda^{2}}{8}+\Lambda M} \sqrt{u+\frac{\Lambda^{2}}{8}-\Lambda M}
\end{aligned}
$$


It has singularities at the zeroes of the discriminant

$$
\Delta=\frac{1}{2^{16}} \Lambda^{4}\left(\Lambda^{2}+8 M^{2}-8 u\right)^{2}\left(\left(\Lambda^{2}+8 u\right)^{2}-64 \Lambda^{2} M^{2}\right),
$$

i.e. at

$$
u_{1}=-M \Lambda-\frac{\Lambda^{2}}{8}, \quad u_{2}=M \Lambda-\frac{\Lambda^{2}}{8}, \quad u_{3}=M^{2}+\frac{\Lambda^{2}}{8} .
$$

The periods $a$ and $a_{D}$ for this curve were explicitly computed in [11]. $a_{D}$ is defined as an integral over the cycle $\gamma_{2}$ surrounding $e_{1}$ and $e_{2}$, whereas $a$ on the cycle $\gamma_{1}$ surrounding $e_{2}$ and $e_{3}$. The cycle $\gamma_{1}$ picks also a pole of the one-form $\lambda$ whose residue is $M / \sqrt{2}$.

One of the salient aspects of this theory is the occurrence of an Argyres-Douglas [12] superconformal fixed point [13]. This arises when some zeroes of $\Delta$ coincide. Then, at the singularity, $e_{1}, e_{2}$ and $e_{3}$ get together and the Riemann surface develops a cusp. From (3.13), we see that this occurs at

$$
\Lambda=2 M
$$

As shown below, this represents nothing but the critical point of a second-order phase transition.

\section{Strong coupling phase $\Lambda>2 M$}

The saddle-point equation is

$$
\frac{\partial S(a, M)}{\partial a}=0 \longrightarrow \operatorname{Im}\left(\frac{\partial \mathscr{F}}{\partial a}\right)=\operatorname{Im}\left(a_{D}\right)=0 .
$$

The behavior of $a_{D}$ was examined in detail in [11] and can be understood by looking at the above expressions for $e_{1}, e_{2}, e_{3}$. In the integration region, $a_{D}$ is purely imaginary. The equation $a_{D}=0$ then requires that $e_{1} \rightarrow e_{2}$. This is the singularity with

$$
u_{3}=M^{2}+\frac{1}{8} \Lambda^{2} .
$$

More precisely, this gives $e_{1}=e_{2}$ provided $\Lambda>2 M$.

The behavior of the saddle-point $a$ at the singularity, $e_{1}=e_{2}$ is shown in fig. $1 \mathrm{~b}$. We have used the exact expressions in terms of elliptic integrals given in [11] (this picks the specific branch where $a\left(u_{3}\right)$ is real). Importantly,

$$
\lim _{M \rightarrow \frac{\Lambda}{2}} a=\frac{M}{\sqrt{2}} .
$$

This is a consequence of the fact that at this point $e_{2} \rightarrow e_{3}$ and the period integral over $\gamma_{1}$ vanishes. When $M \rightarrow \Lambda / 2$, the integral defining the partition function is dominated by a saddle point located precisely at the point where a component of the hypermultiplet becomes massless. From fig. $1 \mathrm{~b}$ we see that the value of $a$ increases from a non-zero value $a=\frac{\Lambda}{\sqrt{2} \pi}$ at $M=0$, until it hits the singularity at $M \rightarrow \Lambda / 2$. As long as $M<\Lambda / 2$, the free energy will be given by $F=-R^{2} \operatorname{Re}\left(4 \pi i \mathscr{F}\left(a^{*}\right)\right)$, where $a^{*}$ is the value of $a$ at $u=u_{3}$. At the critical point, the theory is described by an interacting superconformal theory, whose spectrum of scaling dimensions was discussed in [13].

The free energy is thus completely determined in the strong coupling phase $\Lambda>2 M$ in terms of the prepotential as a function of $M / \Lambda$, computed by sitting on the $u=u_{3}$ singularity. 


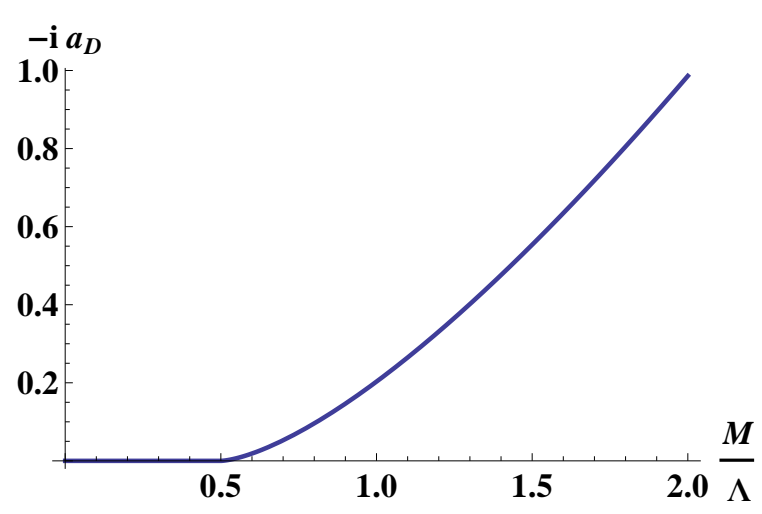

(a)

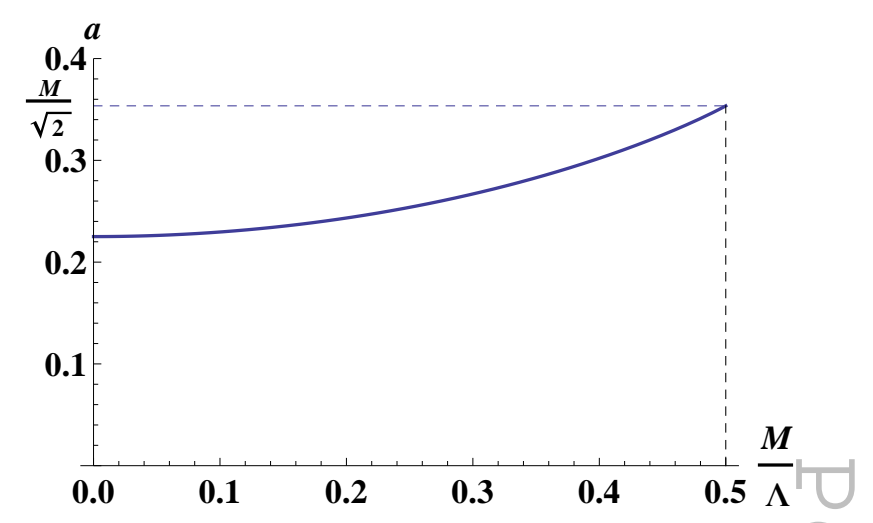

(b)

Figure 1: a) $-i a_{D}$ as a function of $M / \Lambda$ at the singularity $u=M^{2}+\frac{1}{8} \Lambda^{2}$. It vanishes identically for $M<\Lambda / 2$, showing that, in this regime, the singularity $u=u_{3}$ corresponds to a saddle point in the partition function. $\mathrm{b}$ ) $a$ as a function of $M / \Lambda$ at the same singularity.

\section{Weak coupling phase $\Lambda<2 M$}

When $\Lambda<2 M$, then, at $u=u_{3}$ one has $e_{2}=e_{3}$ and $a_{D} \neq 0$. Figure 1a shows a plot of $-i a_{D}$ as a function of $\Lambda / M$, which confirms that $a_{D} \neq 0$ when $M>\Lambda / 2$. This means that the singular point $u=u_{3}$ no longer represents a saddle-point of the integral in the weak coupling regime $\Lambda<2 M$.

Is there any saddle-point dominating the partition function integral in the weak coupling phase $\Lambda<2 M$ ? Above we were looking for saddle-points occurring in the region where $a$ is real and $a_{D}$ is purely imaginary (this was also the assumption in the discussion of [4], which only found the saddle point that computes the integral in the strong coupling phase). In this case, the saddlepoint equation $\operatorname{Im}\left(a_{D}\right)=0$ implies $a_{D}=0$, and we found that there is no saddle with $a_{D}=0$ when $\Lambda<2 M$. Let us now consider more general solutions to the equation

$$
\operatorname{Im}\left(\frac{\partial \mathscr{F}}{\partial a}\right)=\operatorname{Im}\left(a_{D}\right)=0
$$

In the weak coupling phase, $\Lambda<2 M$, one finds that this is satisfied at the singular point

$$
u \rightarrow u_{2}=M \Lambda-\frac{\Lambda^{2}}{8}
$$

At this point, $e_{1} \rightarrow e_{3}$. This represents the dyon singularity

$$
a-\frac{M}{\sqrt{2}}-a_{D}=0 \text {. }
$$

Substituting $u \rightarrow u_{2}$ in the action, we determine the free energy in the weak coupling phase $\Lambda<2 M$.

\section{Free energy and critical behavior}

We can now compute the free energy and its derivatives in the full range $0<\Lambda / M<\infty$. The free energy is obtained as $F=-R^{2} \operatorname{Re}(4 \pi i \mathscr{F})$, where the prepotential can be computed from the integral

$$
\mathscr{F}(u)-\mathscr{F}\left(u_{0}\right)=\int_{u_{0}}^{u} d u a_{D}(u) \partial_{u} a(u) \text {. }
$$


where $u_{0}$ is any generic point on the real line. For $a(u), a_{D}(u)$ one may use the expressions in terms of elliptic functions given in [11]. However, here we do not need the explicit expressions. Here we are interested in the critical behavior of the free energy near the critical point $\Lambda=2 M$. Thus we are interested in the possible discontinuities of the derivatives of the free energy at the critical point. Consider the difference

$$
\left.\Delta F \equiv F\right|_{\Lambda=2 M+\varepsilon}-\left.F\right|_{\Lambda=2 M-\varepsilon}, \quad \varepsilon>0, \quad \varepsilon<<M .
$$

This is obtained as

$$
\Delta F=-R^{2} \operatorname{Re}\left(4 \pi i\left(\mathscr{F}\left(u_{3}\right)-\mathscr{F}\left(u_{2}\right)\right)\right)
$$

with

$$
\mathscr{F}\left(u_{3}\right)-\mathscr{F}\left(u_{2}\right)=\int_{u_{0}}^{u_{3}} d u a_{D}(u) \partial_{u} a(u)-\int_{u_{0}}^{u_{2}} d u a_{D}(u) \partial_{u} a(u),
$$

where the first term is understood to be evaluated at $\Lambda=2 M+\varepsilon$ and the second term, at $\Lambda=2 M-\varepsilon$. Note that $u_{2} \rightarrow u_{3}$ as $\Lambda \rightarrow 2 M$. Then

$$
\begin{aligned}
\partial_{\Lambda} \mathscr{F}\left(u_{3}\right)-\partial_{\Lambda} \mathscr{F}\left(u_{2}\right) & =\left.\left(\partial_{\Lambda} u_{3}\right) a_{D}\left(u_{3}\right) \partial_{u} a(u)\right|_{u=u_{3}}-\left.\left(\partial_{\Lambda} u_{2}\right) a_{D}\left(u_{2}\right) \partial_{u} a(u)\right|_{u=u_{2}} \\
& +\int_{u_{0}}^{u_{3}} d u \partial_{\Lambda}\left(a_{D}(u) \partial_{u} a(u)\right)-\int_{u_{0}}^{u_{2}} d u \partial_{\Lambda}\left(a_{D}(u) \partial_{u} a(u)\right) .
\end{aligned}
$$

Hence

$$
\begin{aligned}
\partial_{\Lambda} \mathscr{F}\left(u_{3}\right)-\partial_{\Lambda} \mathscr{F}\left(u_{2}\right) & =-\left.\frac{M}{2}\left(a\left(u_{2}\right)-\frac{M}{\sqrt{2}}\right) \partial_{u} a(u)\right|_{u=u_{2}} \\
& +\int_{u_{0}}^{u_{3}} d u \partial_{\Lambda}\left(a_{D}(u) \partial_{u} a(u)\right)-\int_{u_{0}}^{u_{2}} d u \partial_{\Lambda}\left(a_{D}(u) \partial_{u} a(u)\right)
\end{aligned}
$$

where we used $a_{D}\left(u_{3}\right)=0$ in the first term (which is to be evaluated at $\Lambda=2 M+\varepsilon$ ) and $a_{D}\left(u_{2}\right)=$ $a\left(u_{2}\right)-\frac{M}{\sqrt{2}}$ in the second term (evaluated at $\Lambda=2 M-\varepsilon$ ). Since, as $\Lambda \rightarrow 2 M, u_{2} \rightarrow u_{3}, a \rightarrow M / \sqrt{2}$ and $\partial_{u} a, a_{D}$ are regular at the critical point, we find that

$$
\left.\left(\partial_{\Lambda} \mathscr{F}\left(u_{3}\right)-\partial_{\Lambda} \mathscr{F}\left(u_{2}\right)\right)\right|_{\varepsilon \rightarrow 0} \rightarrow 0
$$

Therefore the first derivative of the free energy with respect to the coupling $\Lambda$ is continuous at $\Lambda=2 M$. Computing the jump in the second derivative of the prepotential, we now find a nonvanishing contribution

$$
\left.\left(\partial_{\Lambda}^{2} \mathscr{F}\left(u_{3}\right)-\partial_{\Lambda}^{2} \mathscr{F}\left(u_{2}\right)\right)\right|_{\varepsilon \rightarrow 0}=-\left.\frac{M}{2} \partial_{\Lambda} a\left(u_{2}\right) \partial_{u} a(u)\right|_{u=u_{2}} \neq 0
$$

From the explicit expressions, we also find $\left.\operatorname{Im}\left[\partial_{\Lambda} a\left(u_{2}\right) \partial_{u} a(u)\right]\right|_{u=u_{2}} \neq 0$ at $\Lambda=2 M-\varepsilon$. Thus, at the critical point $\Lambda=2 M$, the free energy has a discontinuity in the second derivative, implying a second-order phase transition.

This may be compared with the analogous phase transition occurring in the large $N$ SQCD model, [14, 15], which is, instead, third order. This large $N$ phase transition can also be described in terms of the Seiberg-Witten curve [16]. 
In conclusion, the SQCD $S U(2)$ theory with two flavors has a phase transition of a similar nature as the large $N$ phase transition found in SQCD with $N_{f}<2 N$ flavors discussed in [14, 15]. Just as in the transitions of $[14,15]$, the discontinuous behavior is related to the contribution of massless hypermultiplets to the free energy at the critical point. Unlike the large $N$ phase transitions of $[14,15]$, where instantons are suppressed and played no role, here the phase transition is dominated by instantons. We also note that the Argyres-Douglas superconformal point of [13] represents the critical point of these transitions.

\section{Large $N$ limit of superconformal field theories}

\section{1 $\mathscr{N}=4 S U(N)$ SYM on $\mathbb{S}^{4}$}

The partition function for this theory is particularly simple because instantons do not contribute and the one-loop determinant cancels completely between the $\mathscr{N}=2$ vector multiplet and the $\mathscr{N}=2$ adjoint hypermultiplet. The resulting partition function is described by the Gaussian matrix model:

$$
Z_{\mathscr{N}=4}=\int d^{N-1} a \prod_{i<j}\left(a_{i}-a_{j}\right)^{2} e^{-\frac{8 \pi^{2}}{g^{2}} \sum_{i} a_{i}^{2}}, \quad \sum_{i=1}^{N} a_{i}=0 .
$$

In the large $N$ limit, this integral can be computed by saddle-point techniques. The saddle-point equations $\partial_{a_{i}} S=0$ lead to the system of coupled $N-1$ algebraic equations

$$
\frac{8 \pi^{2}}{g^{2}}=\sum_{j \neq i}\left(a_{j}-a_{i}\right)^{-1} .
$$

The large $N$ limit can be conveniently described as usual by going to the continuum limit by introducing the eigenvalue density

$$
\rho(x)=\frac{1}{N} \sum_{i=1}^{N} \delta\left(x-a_{i}\right)
$$

Then the saddle-point equations are replaced by the singular integral equation:

$$
f_{-\mu}^{\mu} d y \frac{\rho(y)}{x-y}=\frac{8 \pi^{2}}{\lambda} x
$$

The solution is given by

$$
\rho(x)=\frac{8 \pi}{\lambda} \sqrt{\frac{\lambda}{4 \pi^{2}}-x^{2}} .
$$

Thus eigenvalues have a semi-circular distribution (the Wigner semi-circle). This can be proved by choosing a contour around the cut from $-\mu$ to $\mu$ and computing the residue at infinity. Having the eigenvalue density, we can compute the VEV of the 1/2 supersymmetric circular Wilson loop operator:

$$
W(C)=\operatorname{Tr} P \exp \left[\int_{C} d s\left(A_{\mu}(x) \dot{x}^{\mu}+i \Phi|\dot{x}|\right)\right]
$$

This localizes to

$$
\langle W\rangle=\left\langle\sum_{j=1}^{N} e^{2 \pi a_{j}}\right\rangle=\int_{-\mu}^{\mu} d x \rho(x) e^{2 \pi x}=\frac{2}{\sqrt{\lambda}} I_{1}(\sqrt{\lambda}) .
$$

This represents the leading result in the $1 / N$ expansion $[17,18]$. 


\subsection{Correlation functions of chiral primary operators}

A particularly interesting sector of $\mathscr{N}=2$ superconformal theories (SCFT's) is that originated from primary operators annihilated by all supercharges of one chirality, known as chiral primary operators (CPO's). In superspace language, the scaling dimension $\Delta$ of chiral primaries are bottom components of $\mathscr{N}=2$ chiral superfields. The case of $\Delta=2$ is particularly interesting, as the integrated top component of the multiplet defines an exactly marginal operator. Hence, chiral superfields with $\Delta=2$ parametrize the conformal manifold associated to the SCFT. Moreover, the 2-point function of such top components defines the Zamolodchikov metric on the conformal manifold. Because of supersymmetry, it turns out that such metric can be read from the correlators of the CPO's. One may choose a basis where the 2-point functions of CPO's on $\mathbb{R}^{4}$ are of the form

$$
\left\langle O_{n}(x) \bar{O}_{\bar{m}}(0)\right\rangle_{\mathbb{R}^{4}}=\frac{G_{n \bar{m}}}{|x|^{2 \Delta_{n}}} \delta_{\Delta_{n} \Delta_{\bar{m}}} .
$$

The metric $G_{n \bar{m}}$ encodes all the essential data and it is the main object of interest. These correlation functions can be computed exactly in any $\mathscr{N}=2$ superconformal gauge theory by a construction based on localization recently developed by [5]. The idea exploits the fact that scaling dimension of chiral primaries are bottom components of $\mathscr{N}=2$ chiral superfields. This permits to deform the action by preserving $\mathscr{N}=2$ superconformal invariance. The method can be used to compute correlators of the form

$$
\left\langle\mathscr{O}_{n_{1}}\left(x_{1}\right) \cdots \mathscr{O}_{n_{n}}\left(x_{n}\right){\overline{O^{\prime}}}_{m}(y)\right\rangle_{\mathbb{R}^{4}}
$$

Furthermore, due to supersymmetry, correlators on $\mathbb{R}^{4}$ satisfy that [19]

$$
\begin{gathered}
\left\langle\mathscr{O}_{n_{1}}\left(x_{1}\right) \cdots \mathscr{O}_{n_{n}}\left(x_{n}\right){\overline{O^{\prime}}}_{m}(y)\right\rangle_{\mathbb{R}^{4}}=\left\langle O_{n}\left(x_{1}\right){\overline{O^{\prime}}}_{m}(y)\right\rangle_{\mathbb{R}^{4}}, \\
O_{n}\left(x_{1}\right) \equiv \mathscr{O}_{n_{1}}\left(x_{1}\right) \cdots \mathscr{O}_{n_{n}}\left(x_{n}\right)
\end{gathered}
$$

for any number of chiral primary operators and one anti-chiral primary.

To compute these correlators, the idea is to add a source $\tau_{n}$ for all CPO's, obtaining in this way a deformed partition function. In [6], correlators in $\mathscr{N}=4$ super Yang-Mills theory have been computed by solving the deformed matrix model in the large $N$ limit. We now describe this calculation. In this limit, the set of CPO's dramatically simplifies as only single-trace operators contribute. Thus, the operators in the chiral ring are of the form $O_{n}^{\mathbb{R}^{4}}=\operatorname{Tr} \phi^{n}$, being $\phi$ one of the complex scalars in the theory (the scalar field of the vector multiplet when the theory is viewed as an $\mathscr{N}=2$ theory). Localization sets $\operatorname{Tr} \phi^{n}=\sum_{i=1}^{N} a_{i}^{n}$. Thus, for $\mathscr{N}=4 U(N)$ super Yang-Mills theory, one has to consider the deformed matrix model

$$
\mathscr{Z}=\int d^{N} a \prod_{i<j}\left(a_{i}-a_{j}\right)^{2}\left|e^{i \sum_{n=1}^{N} \pi^{n / 2} \tau_{n} \sum_{i}\left(a_{i}\right)^{n}}\right|^{2},
$$

where $\tau_{2}=\tau$ is the Yang-Mills coupling (2.10).

Two-point correlators are then computed by

$$
\frac{1}{\mathscr{Z}\left(\tau_{n}, \bar{\tau}_{\bar{n}}\right)} \partial_{\tau_{n}} \partial_{\bar{\tau}_{\bar{m}}} \mathscr{Z}\left(\tau_{n}, \bar{\tau}_{\bar{n}}\right)=\int_{\mathbb{S}^{4}} d^{4} x \sqrt{g(x)} \int_{\mathbb{S}^{4}} d^{4} y \sqrt{g(y)}\left\langle O_{n}(x) \bar{O}_{\bar{m}}(y)\right\rangle_{S^{4}} .
$$


Due to the conformal anomaly, on the $\mathbb{S}^{4}$ there is a highly non-trivial operator mixture. This is expected, since the $\mathbb{S}^{4}$ theory preserves the supergroup $\operatorname{osp}(2 \mid 4)$, which contains the $S U(2)_{R}$ symmetry but breaks the $U(1)_{R}$ symmetry. Thus, mixtures among different CPO's are possible. Indeed, denoting by $R$ the radius of the $\mathbb{S}^{4}$, a given operator $O_{\Delta}$ of dimension $\Delta$ on $\mathbb{R}^{4}$, when mapped into $\mathbb{S}^{4}$, generically mixes with all operators with lower dimensions in steps of 2 , that is

$$
O_{\Delta}^{\mathbb{R}^{4}} \rightarrow O_{\Delta}^{\mathbb{S}^{4}}+\alpha_{\Delta}^{(2)} \frac{1}{R^{2}} O_{\Delta-2}^{\mathbb{S}^{4}}+\alpha_{\Delta}^{(4)} \frac{1}{R^{4}} O_{\Delta-4}^{\mathbb{S}^{4}}+\cdots
$$

In mapping the $\mathbb{S}^{4}$ computation back into the $\mathbb{R}^{4}$, the operator mixture must be disentangled. This can be accomplished by a Gram-Schmidt orthogonalization procedure. This method was recently used to compute large $N$ correlation functions in superconformal field theories in $[6,7,20]$. In what follows we set $R=1$.

Returning to the calculation of correlation functions, we need to solve a matrix model with a potential. Consider, for example, the calculation of correlation function for even operators $\operatorname{Tr} \phi^{2 n}$. By virtue of (4.11), they do not mix with odd operators. Including only even deformations, it is useful to redefine the potential as

$$
V=\sum_{n=1}^{n_{0}} g_{2 n} x^{2 n}, \quad n_{0} \equiv[N / 2]
$$

Since the potential is invariant under reflection symmetry, we can assume that eigenvalues will condense in a cut $(-\mu, \mu)$. The solution for the large $N$ eigenvalue distribution of a matrix model with an arbitrary even potential is well known,

$$
\rho(z)=\frac{1}{4 \pi^{2}} \sqrt{\mu^{2}-z^{2}} f_{-\mu}^{\mu} d x \frac{V^{\prime}(x)}{\sqrt{\mu^{2}-x^{2}}(z-x)} .
$$

In the present case, we get

$$
\rho(z)=\frac{1}{2 \pi^{2}} \sqrt{\mu^{2}-z^{2}} \sum_{n=1}^{n_{0}} n g_{2 n} f_{-\mu}^{\mu} d x \frac{x^{2 n-1}}{\sqrt{\mu^{2}-x^{2}}(z-x)} .
$$

The integral can be computed by choosing a contour that surrounds the cut $(-\mu, \mu)$ and computing the residue at infinity. This gives the formula

$$
f_{-\mu}^{\mu} d x \frac{x^{2 n-1}}{\sqrt{\mu^{2}-x^{2}}(z-x)}=2 \pi \sum_{k=0}^{n-1} b_{k} z^{2 n-2 k-2} \mu^{2 k} .
$$

Therefore, we obtain

$$
\rho(x)=\left(\sum_{k=0}^{n_{0}-1} q_{k} z^{2 k}\right) \sqrt{\mu^{2}-z^{2}},
$$

with

$$
\begin{gathered}
q_{k}=\frac{1}{\pi} \sum_{n=k+1}^{n_{0}} n b_{n-k-1} g_{2 n} \mu^{2 n-2 k-2} \\
b_{k} \equiv \frac{1}{\sqrt{\pi}} \frac{\Gamma(k+1 / 2)}{k !}
\end{gathered}
$$


Let us now consider the normalization condition,

$$
\int_{-\mu}^{\mu} d x \rho(x)=1
$$

Again, by residues

$$
\int_{-\mu}^{\mu} d z z^{2 k} \sqrt{\mu^{2}-z^{2}}=\pi \sigma_{k+1} \mu^{2 k+2},
$$

where

$$
\sigma_{k} \equiv \frac{1}{2 \sqrt{\pi}} \frac{\Gamma(k-1 / 2)}{k !}
$$

Hence, normalization implies

$$
\pi \sum_{k=0}^{n_{0}-1} q_{k} \sigma_{k+1} \mu^{2 k+2}=1 .
$$

Using the expression for $c_{k}$, this becomes

$$
\sum_{n=1}^{n_{0}} n b_{n} g_{2 n} \mu^{2 n}=1
$$

where we used the identity

$$
\sum_{k=0}^{n-1} \sigma_{k+1} b_{n-k-1}=b_{n}
$$

In order to compute the connected correlators, we need to compute the $g_{2 n}$ derivatives of $F=-\ln Z$. In particular, for two point functions, we need to compute the matrix of second derivatives of $F$. We begin with the formula

$$
\partial_{g_{2 n}} F=\int_{-\mu}^{\mu} d z z^{2 n} \rho(z)
$$

This gives

$$
\partial_{g_{2 n}} F=\pi \sum_{k=0}^{n_{0}-1} q_{k} \sigma_{k+n+1} \mu^{2 k+2 n+2}=\sum_{m=1}^{n_{0}} d_{m, n} g_{2 m} \mu^{2 m+2 n}
$$

with

$$
d_{m, n} \equiv m \sum_{k=0}^{m-1} b_{m-k-1} \sigma_{k+n+1}=\frac{(2 m) ! \Gamma\left(n+\frac{1}{2}\right)}{4^{m} \sqrt{\pi}(m+n) n !(m-1) !^{2}} .
$$

Before taking the second derivative, we note that from this formula we can already obtain the VEV of the $\operatorname{Tr} \phi^{2 n}$. We must set $g_{2 n}=0$, with $n>1$ and

$$
g_{2}=\frac{8 \pi^{2}}{\lambda}
$$

This gives

$$
\left\langle\operatorname{Tr} \phi^{2 r}\right\rangle_{\mathbb{S}^{4}}=\left.\partial_{g_{2} r} F\right|_{g_{2 r}=0, r>1}=N\left(\frac{\lambda}{4 \pi^{2}}\right)^{r} \frac{\Gamma\left(r+\frac{1}{2}\right)}{\sqrt{\pi}(r+1) !}=N\left(\frac{\lambda}{4 \pi^{2}}\right)^{r} \frac{(2 r) !}{4^{r} r !(r+1) !},
$$

for $r=1,2, \ldots$ Similarly, one can obtain that $\left\langle\operatorname{Tr} \phi^{2 r+1}\right\rangle_{\mathbb{S}^{4}}=0$. 
Next, we compute the second derivative of the free energy,

$$
\partial_{g_{2 m}} \partial_{g_{2 n}} F=\sum_{k=1}^{n_{0}}(2 k+2 n) d_{k, n} g_{2 k} \mu^{2 k+2 n-1} \frac{d \mu}{d g_{2 m}}+d_{m, n} \mu^{2 m+2 n} .
$$

To compute $d \mu / d g_{2 m}$, we use the normalization condition. After differentiation, we must set all $g_{2 n}=0$, with $n>1$. We get

$$
\frac{d \mu}{d g_{2 k}}=-\frac{k b_{k} \mu^{2 k-1}}{g_{2}}=-\frac{1}{2} k b_{k} \mu^{2 k+1}
$$

where we used

$$
\mu^{2}=\frac{2}{g_{2}}=\frac{\lambda}{(2 \pi)^{2}}
$$

Therefore

$$
\left.\partial_{g_{2 m}} \partial_{g_{2 n}} F\right|_{g_{2 k>2}=0}=\left(d_{m, n}-(2+2 n) d_{1, n} m b_{m}\right) \mu^{2 m+2 n} .
$$

After some simple algebra, we obtain

$$
\left\langle O_{2 m} \bar{O}_{2 n}\right\rangle_{\mathbb{S}^{4}}=\partial_{g_{2 m}} \partial_{g_{2 n}} F=-\left(\frac{\lambda}{4 \pi^{2}}\right)^{m+n} \frac{\Gamma\left(m+\frac{1}{2}\right) \Gamma\left(n+\frac{1}{2}\right)}{\pi(m+n) \Gamma(m) \Gamma(n)},
$$

where we have defined the VEV-less operators,

$$
O_{n}^{\mathbb{S}^{4}}=\left.\operatorname{Tr} \phi^{n}\right|_{\mathbb{S}^{4}}-\left\langle\operatorname{Tr} \phi^{n}\right\rangle_{\mathbb{S}^{4}} \mathbf{1}
$$

The correlators (4.32) look very different from the result obtained by explicit calculation by Feynman diagrams. Due to a non-renormalization theorem, in $\mathscr{N}=4 \mathrm{SYM}$ one finds the exact result [21]

$$
\left\langle O_{n}^{\mathbb{R}^{4}}(0) \bar{O}_{\bar{m}}^{\mathbb{R}^{4}}(x)\right\rangle_{\mathbb{R}^{4}}=\frac{\delta_{n \bar{m}}}{|x|^{2 \Delta_{n}}} \frac{\Delta_{n} \lambda^{\Delta_{n}}}{(2 \pi)^{2 \Delta_{n}}} .
$$

Instead, we are getting non-vanishing two-point functions between operators of different dimensions, whereas the explicit calculation shows that the 2-point function (4.34) vanishes unless the operators have the same dimension. The key point is the mixture that occurs on $\mathbb{S}^{4}$. By running the Gram-Schmidt procedure (and including also the odd-odd correlators), we find the orthogonal basis

$$
\begin{aligned}
& O_{1}^{S^{4}} ; \\
& O_{2}^{S^{4}} ; \\
& O_{3}^{S^{4}}-\frac{3 \lambda}{(4 \pi)^{2}} O_{1}^{S^{4}} ; \\
& O_{4}^{S^{4}}-\frac{4 \lambda}{(4 \pi)^{2}} O_{2}^{S^{4}} ; \\
& O_{5}^{S^{4}}-\frac{5 \lambda}{(4 \pi)^{2}} O_{3}^{S^{4}}+\frac{5 \lambda^{2}}{(4 \pi)^{4}} O_{1}^{S^{4}} ; \\
& O_{6}^{S^{4}}-\frac{6 \lambda}{(4 \pi)^{2}} O_{4}^{S^{4}}+\frac{9 \lambda^{2}}{(4 \pi)^{4}} O_{2}^{S^{4}} ;
\end{aligned}
$$


In the new basis, we find the expected result (4.34).

Here we have checked up to $O_{6}$. Clearly, it would be desirable to find the new orthogonal basis by extending (4.35) to all operators, to fully disentangle the operator mixing on $\mathbb{S}^{4}$. By inspection (and luck), we find the remarkable formula,

$$
\begin{aligned}
& O_{n}^{\mathbb{R}^{4}}=2\left(\frac{\lambda}{(4 \pi)^{2}}\right)^{\frac{n}{2}} \operatorname{Tr}\left[T_{n}\left(\frac{2 \pi}{\sqrt{\lambda}} \phi\right)\right], \quad n \neq 2, \\
& O_{2}^{\mathbb{R}^{4}}=\frac{\lambda}{(4 \pi)^{2}}\left(2 \operatorname{Tr}\left[T_{2}\left(\frac{2 \pi}{\sqrt{\lambda}} \phi\right)\right]+1\right)
\end{aligned}
$$

Using this formula, one can easily prove in full generality (4.34) from the matrix model on $\mathbb{S}^{4}$ [7].

\subsection{Correlation functions involving Wilson loop operators}

Having found a closed analytic formula for the operator mixing on $\mathbb{S}^{4}$, we can now compute other correlation functions. Consider first the VEV of the 1/2 BPS circular Wilson loop operator: Expanding the exponential, it follows that

$$
\langle W\rangle_{\mathbb{S}^{4}}=\sum_{k=0}^{\infty} \frac{(2 \pi)^{n}}{n !}\left\langle\operatorname{Tr} \phi^{n}\right\rangle .
$$

Substituting (4.27) into (4.37), we find

$$
\langle W\rangle_{\mathbb{S}^{4}}=\frac{2}{\sqrt{\lambda}} I_{1}(\sqrt{\lambda})
$$

where $I_{n}$ denotes, as usual, the modified Bessel function of the first kind. This reproduces the familiar formula $[17,18]$ for the VEV of the circular Wilson loop discussed in section 4.1.

Next, consider

$$
\left\langle O_{n}^{\mathbb{R}^{4}} W\right\rangle_{\mathbb{S}^{4}}=\sum_{r=0}^{\infty} \frac{(2 \pi)^{r}}{r !}\left\langle O_{n}^{\mathbb{R}^{4}} \operatorname{Tr} \bar{\phi}^{r}\right\rangle_{\mathbb{S}^{4}}
$$

This has been computed by Giombi and Pestun from a two-matrix model proposal [22]. Here we shall rederive this result by writing $O_{n}^{\mathbb{R}^{4}}$ in terms of $\operatorname{Tr} \phi^{n}$ and using our general formula given by the Chebyshev polynomials. Using the explicit expansion of the Chebyshev polynomial, after some algebra, we find

$$
\begin{aligned}
\left\langle O_{2 n}^{\mathbb{R}^{4}} W\right\rangle_{\mathbb{S}^{4}} & =2 n\left(\frac{\lambda}{8 \pi}\right)^{2 n} \sum_{r=0}^{\infty} \frac{1}{r !(r+2 n) !}\left(\frac{\lambda}{4}\right)^{r} \\
& =2 n\left(\frac{\lambda}{(4 \pi)^{2}}\right)^{n} I_{2 n}(\sqrt{\lambda})
\end{aligned}
$$

which is in exact agreement with the results of Giombi and Pestun [22], this time obtained from first principles, namely by direct evaluation of the correlators on $\mathbb{S}^{4}$ using localization and then going to the orthogonal basis. 


\section{4 $\mathscr{N}=2$ Superconformal QCD}

The previous method to compute correlators can be extended to other $\mathscr{N}=2$ superconformal field theories. In perturbation theory, one can express correlators of any $\mathscr{N}=2 S C F$ in terms of correlators of the $\mathscr{N}=4$ theory. As an example, consider $\mathscr{N}=2$ superconformal QCD, that is $\mathscr{N}=2 \mathrm{SYM}$ with gauge group $S U(N)$ and $2 N$ fundamental (flavor) hypermultiplets. The deformed partition function is given by

$$
\begin{gathered}
Z_{\mathscr{N}=2}=\int d^{N-1} a \Delta(a) \frac{\prod_{i<j} H\left(a_{i}-a_{j}\right)^{2}}{\prod_{i} H\left(a_{i}\right)^{2 N}}\left|e^{-2 \pi \operatorname{Im} \tau_{Y M} \sum a_{i}^{2}}\right| \mathscr{Z}_{\text {inst }}, \\
H(x)=\prod_{n=1}^{\infty}\left(1+\frac{x^{2}}{n^{2}}\right)^{n^{2}} e^{-\frac{x^{2}}{n}} .
\end{gathered}
$$

We shall consider the perturbative expansion in the zero instanton number sector, so we set $\mathscr{Z}_{\text {inst }} \rightarrow$ 1. Perturbation series is obtained by expanding the one-loop factor in powers of $a_{i}$. We use

$$
\ln H(x)=-\sum_{n=2}^{\infty}(-1)^{n} \frac{\zeta(2 n-1)}{n} x^{2 n} .
$$

Now we can expand $\mathscr{Z}_{\mathscr{N}=2}$ as

$$
\begin{aligned}
Z_{\mathscr{N}=2}= & Z_{\mathscr{N}=4}\left\{1-3 \zeta(3)\left\langle\operatorname{Tr} \phi^{2} \operatorname{Tr} \bar{\phi}^{2}\right\rangle_{S^{4}}^{\mathscr{N}=4}\right. \\
& \left.-\frac{2}{3} \zeta(5)\left(10\left\langle\operatorname{Tr} \phi^{3} \operatorname{Tr} \bar{\phi}^{3}\right\rangle_{S^{4}}^{\mathscr{N}=4}-15\left\langle\operatorname{Tr} \phi^{4} \operatorname{Tr} \bar{\phi}^{2}\right\rangle_{S^{4}}^{\mathscr{N}=4}\right)+\cdots\right\}
\end{aligned}
$$

where $Z_{\mathscr{N}=4}$ is the $S U(N) \mathscr{N}=4 \mathrm{SYM}$ partition function and $\left\langle\operatorname{Tr} \phi^{n} \operatorname{Tr} \bar{\phi}^{m}\right\rangle_{S^{4}}^{\mathscr{N}=4}$ refers to the 2point function of the $\operatorname{Tr} \phi^{n}, \operatorname{Tr} \bar{\phi}^{m}$ operators in the $\mathscr{N}=4 \mathrm{SYM}$ matrix model on the $\mathbb{S}^{4}$. In this way we write the partition function for $\mathscr{N}=2$ superconformal QCD solely in terms of quantities in $\mathscr{N}=4 \mathrm{SYM}$ at arbitrary $N$.

Let us now consider correlators for CPO's. The special case of correlators for $\operatorname{Tr} \phi^{2}$ is simple, as these insertions arise from derivatives of the YM coupling $\tau_{Y M}$. One finds [7]

$$
\left\langle\operatorname{Tr} \phi^{2} \operatorname{Tr} \bar{\phi}^{2}\right\rangle_{\mathbb{R}^{4}}^{\mathscr{N}=2, S U(N)}=\frac{2\left(N^{2}-1\right)}{\pi^{2} \operatorname{Im} \tau_{Y M}^{2}}-\frac{9 \zeta(3)\left(N^{2}-1\right)\left(N^{2}+1\right)}{2 \pi^{4} \operatorname{Im} \tau_{Y M}^{4}}+\cdots,
$$

In the particular case of $S U(2)$ gauge group, this formula reproduces an earlier result given in [19] (see also [5]) and, for $S U(3), S U(4)$, the formula (4.44) also reproduces the expressions given in $[23,5]$.

\section{Conclusions}

- Finite $N$ : One can show the existence of phase transitions in massive $\mathscr{N}=2$ theories by combining the Seiberg-Witten curve and saddle-point techniques. Extending the results of [4], we showed that four-dimensional $\mathscr{N}=2 S U(2)$ SQCD with two massive flavors has a second-order quantum phase transition. Saddle-points occur at singularities of the SeibergWitten curve. As the coupling is increased, it crosses a critical value $\Lambda=2 M$ after which 
the saddle-point jumps to another value, producing a discontinuity in the second derivative of the free energy.

- From the free energy in the weak-coupling phase one could compute the weak-coupling OPE expansion for the full model including instanton contributions, by expanding the free energy in powers of $\Lambda / M$. In particular, this would be interesting in order to have a better understanding of a long-standing question in QCD, concerning the precise manner by which instanton and non-instanton power-like corrections contribute, and how they can be distinguished.

- It would be interesting to understand the phase structure of $\mathscr{N}=2^{*} S U(N)$ theory using the Seiberg-Witten curve, as we here did for $S U(2)$ SQCD. Some hints of a finite $N$ phase transition have been recently found in [24].

- Correlation functions of chiral primaries in $\mathscr{N}=2$ superconformal theories can be calculated using a suitably deformed matrix model.

- For the $\mathscr{N}=4$ theory, the operator mixing on $\mathbb{S}^{4}$ is given by the compact formula (4.36) in terms of Chebyshev polynomials.

- An interesting challenge is to compute the operator mixing coefficients using holographic duality to $A d S_{5} \times S^{5}$.

- The method can be applied to compute correlator functions for other operators, e.g. $\langle W W\rangle$, in many other SCF, including quivers.

\section{References}

[1] N. A. Nekrasov, "Seiberg-Witten Prepotential From Instanton Counting," Adv. Theor. Math. Phys. 7, 831 (2004) [arXiv:hep-th/0206161].

[2] N. Nekrasov and A. Okounkov, "Seiberg-Witten theory and random partitions," arXiv:hep-th/0306238.

[3] V. Pestun, "Localization of gauge theory on a four-sphere and supersymmetric Wilson loops," Commun. Math. Phys. 313, 71 (2012) [arXiv:0712.2824 [hep-th]].

[4] J. G. Russo, “ $\mathscr{N}=2$ gauge theories and quantum phases,” JHEP 1412, 169 (2014) [arXiv:1411.2602 [hep-th]].

[5] E. Gerchkovitz, J. Gomis, N. Ishtiaque, A. Karasik, Z. Komargodski and S. S. Pufu, "Correlation Functions of Coulomb Branch Operators," arXiv:1602.05971 [hep-th].

[6] D. Rodriguez-Gomez and J. G. Russo, "Large N Correlation Functions in Superconformal Field Theories,” JHEP 1606, 109 (2016) doi:10.1007/JHEP06(2016)109 [arXiv:1604.07416 [hep-th]].

[7] D. Rodriguez-Gomez and J. G. Russo, "Operator Mixing in Large N Superconformal Field Theories on $\mathbb{S}^{4}$ and Correlators with Wilson loops,” JHEP, in press arXiv:1607.07878 [hep-th].

[8] Y. Ohta, "Prepotentials of N=2 SU(2) Yang-Mills theories coupled with massive matter multiplets," J. Math. Phys. 38, 682 (1997) [hep-th/9604059]. 
[9] E. D'Hoker, I. M. Krichever and D. H. Phong, "The Effective prepotential of N=2 supersymmetric SU(N(c)) gauge theories," Nucl. Phys. B 489, 179 (1997) [hep-th/9609041].

[10] N. Seiberg and E. Witten, "Monopoles, duality and chiral symmetry breaking in N=2 supersymmetric QCD,” Nucl. Phys. B 431, 484 (1994) [hep-th/9408099].

[11] A. Bilal and F. Ferrari, "The BPS spectra and superconformal points in massive $\mathrm{N}=2$ supersymmetric QCD,” Nucl. Phys. B 516, 175 (1998) [hep-th/9706145].

[12] P. C. Argyres and M. R. Douglas, "New phenomena in SU(3) supersymmetric gauge theory," Nucl. Phys. B 448, 93 (1995) [hep-th/9505062].

[13] P. C. Argyres, M. R. Plesser, N. Seiberg and E. Witten, "New N=2 superconformal field theories in four-dimensions," Nucl. Phys. B 461, 71 (1996) [hep-th/9511154].

[14] J. G. Russo and K. Zarembo, "Massive N=2 Gauge Theories at Large N," JHEP 1311, 130 (2013) [arXiv:1309.1004 [hep-th]].

[15] J. G. Russo and K. Zarembo, "Localization at Large N," arXiv:1312.1214 [hep-th].

[16] J. G. Russo, “Large $N_{c}$ from Seiberg-Witten Curve and Localization,” Phys. Lett. B 748, 19 (2015) [arXiv:1504.02958 [hep-th]].

[17] J. K. Erickson, G. W. Semenoff and K. Zarembo, "Wilson loops in N=4 supersymmetric Yang-Mills theory,” Nucl. Phys. B 582, 155 (2000) [hep-th/0003055].

[18] N. Drukker and D. J. Gross, "An Exact prediction of N=4 SUSYM theory for string theory," J. Math. Phys. 42, 2896 (2001) [hep-th/0010274].

[19] M. Baggio, V. Niarchos and K. Papadodimas, " $\mathrm{tt}^{*}$ equations, localization and exact chiral rings in 4d $\mathscr{N}=2$ SCFTs,” JHEP 1502, 122 (2015) [arXiv:1409.4212 [hep-th]].

[20] M. Baggio, V. Niarchos, K. Papadodimas and G. Vos, "Large-N correlation functions in $\mathscr{N}=2$ superconformal QCD," arXiv:1610.07612 [hep-th].

[21] S. Lee, S. Minwalla, M. Rangamani and N. Seiberg, "Three point functions of chiral operators in D = 4, N=4 SYM at large N,” Adv. Theor. Math. Phys. 2, 697 (1998) [hep-th/9806074].

[22] S. Giombi and V. Pestun, "Correlators of local operators and 1/8 BPS Wilson loops on S**2 from 2d YM and matrix models,” JHEP 1010, 033 (2010) [arXiv:0906.1572 [hep-th]].

[23] M. Baggio, V. Niarchos and K. Papadodimas, "On exact correlation functions in SU(N) $\mathscr{N}=2$ superconformal QCD,” JHEP 1511, 198 (2015) [arXiv:1508.03077 [hep-th]].

[24] T. J. Hollowood and S. P. Kumar, "Partition function of $\mathscr{N}=2^{*}$ SYM on a large four-sphere," JHEP 1512, 016 (2015) [arXiv:1509.00716 [hep-th]]. 